\title{
Choice of the detectors for light impurities plasma studies at W7-X using 'CO Monitor' system
}

\author{
Tomasz Fornal*a, Ireneusz Ksiazek ${ }^{\mathrm{b}}$, Monika Kubkowska ${ }^{\mathrm{a}}$, Jacek Kaczmarczyk ${ }^{\mathrm{a}}$, \\ Rainer Burhenn ${ }^{\mathrm{c}}$ and $\mathrm{W} 7-\mathrm{X}$ team $^{\mathrm{c}}$ \\ ${ }^{a}$ Institute of Plasma Physics and Laser Microfusion, Hery 23 St., 01-497 Warsaw, Poland
bInstitute of Physics, Opole University, Oleska 48 St., 45-052 Opole, Poland
${ }^{c}$ Max-Planck-Institut fürPlasmaphysik, Wendelsteinstrasse 1, 17491 Greifswald, Germany
}

The 'CO Monitor' is a new spectrometer system dedicated for the continuous measurements of line intensities of carbon, oxygen, boron and nitrogen at the fusion plasma experiment Wendelstein 7-X (W7-X). Its main purpose is to deliver constant information about indicated elements with high time resolution (better than $1 \mathrm{~ms}$ ), but low spatial resolution since the line shapes are not going to be investigated. The system consists of four independent channels, each equipped with dispersive element dedicated for measurement of selected line of interest. In order to perform the highest efficiency of the 'CO Monitor' system, it is essential to choose the proper detector type for this task. The considered options are Micro Channel Plates (MCP), Charge-Coupled Device (CCD cameras), Multi Strip Gaseous Chambers (MSGC) and the Gas Electron Multipliers (GEM) detectors. For the second Operational Phase (OP 2) of the W7-X the CCD cameras are the main candidates as a detection system for the 'CO Monitor' purpose due to their high sensitivity and the fact, that they are commercially available. Nevertheless, all other detector types are taken into account for the latter experimental operation when investigations of deuterium plasmas will start.

\section{Keywords: XUV; detectors; Wendelstein 7-X; stellarator}

\section{Introduction}

In magnetically confined fusion plasmas, the measurement of the impurities and their behaviour is a very essential task. Monitoring of their content delivers information about, e.g., plasma composition, overheating, energy radiated from plasma, etc. During the experimental campaigns Operational Phases 1.1 and 1.2 (OP 1.1 and 1.2) on the Wendelstein 7-X stellarator (W7$\mathrm{X})$, there were many diagnostic systems [1-3] dedicated for the measurement of impurity content in the plasma (e.g., X-ray radiation pulse-height analyser - PHA[4], VUV grating spectrometer - HEXOS[5] or XICS/HR$\mathrm{XIS}[6][7])$. The 'CO Monitor' system is a new diagnostic dedicated for the W7-X stellarator which has been designed and is planned to be built and installed before the next operational phase on the W7-X, OP2. This device is an equipment dedicated for the observation of Lyman$\alpha$ lines of light impurities such as carbon (C VI - 3.4 nm), oxygen (O VIII - $1.9 \mathrm{~nm})$, boron $(\mathrm{B} \mathrm{V}-4.9 \mathrm{~nm})$ and nitrogen (N VII - $2.5 \mathrm{~nm}$ ) ('CO Monitor' is a historical name of the system but the diagnostic will measure also $\mathrm{B}$ and N). Fast monitoring of those lines would deliver an information about wall conditioning (oxygen), overheating of plasma facing components (carbon) and the quality of boron layer (boron). The possible malfunction of vacuum system will be indicated by monitoring of oxygen and nitrogen. For the proper machines operation this knowledge is essential, and their constant monitoring would help to improve the operation of the W7-X as well as the future fusion devices.

The system consists of two vacuum chambers with crystal or multilayer mirrors as dispersive elements selected for reflection of particular photon wavelengths corresponding to the respective ions (carbon, oxygen, boron and nitrogen). The chambers are positioned at the elongated
AEK30 port, horizontally one over another with their line of sight crossing at the main magnetic axis of the W7-X (see Fig. 1.).

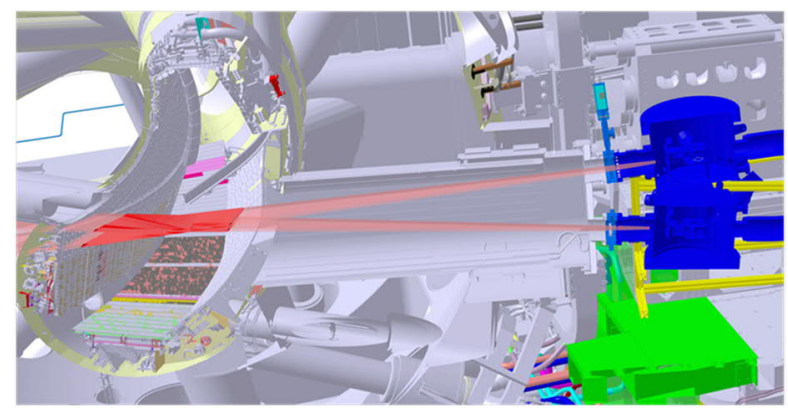

Fig. 1. 'CO Monitors' lines of sight crossing at the main magnetic axis.

The 'CO Monitor' diagnostic system is characterized by its simple construction and is dedicated directly for the fast measurement of selected lines. Its design is based on the Johann geometry providing an energy resolved spectrum. This geometry allows to register simultaneously the radiation intensity in a defined wavelength range. In the case of the 'CO Monitor' each channel registers the wavelength range of the selected spectral line associated with a line core and its far wings (approx. $0.4 \mathrm{~nm}$ ). Thus, the measured line radiation can be corrected by the background level (visible in the far wings of the line) simultaneously. Due to the clear separation of lines in this spectral range, the spectral resolution can be poor. The estimated spectral resolution for the channels equipped with multilayer mirrors is approximately $0.01 \mathrm{~nm} / \mathrm{mm}$. In the case of O VIII $(1.9 \mathrm{~nm})$ channel equipped with TlAP crystal, the resolution is even higher (approx. $0.003 \mathrm{~nm} / \mathrm{mm}$ ). Nevertheless, while the detectors will not be positioned tangentially to the 
Rowland circle, the line shapes are not going to be investigated (see Fig. 2.).

To obtain high count rate with high time resolution (and sufficiently good signal to noise ratio), it is necessary to register sufficiently high flux of XUV quanta. Therefore, the construction of the system should provide quite large solid angle of the input (resigning the spatial resolution).

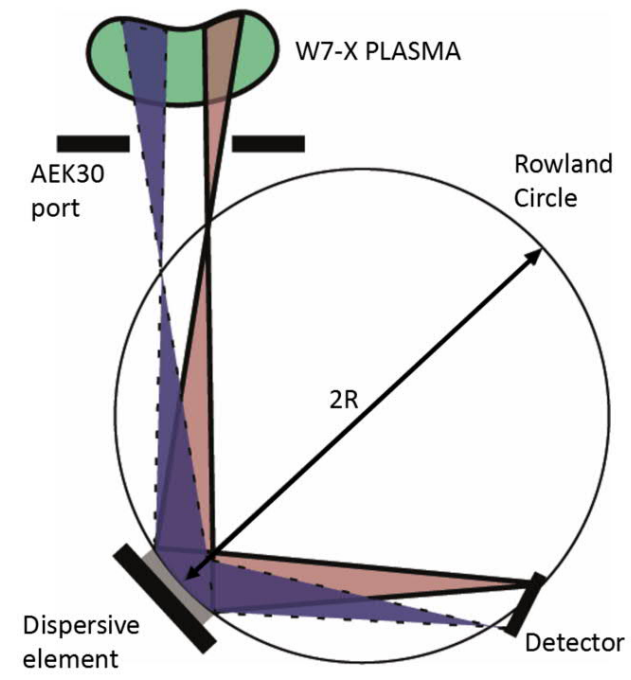

Fig. 2. 'CO Monitor' system principle based on Johann geometry.

For the proper operation of the 'CO Monitor' spectrometer and to obtain a reliable data about the plasma composition, choosing the right detector type is really crucial. Several types of detectors were considered for this application which need to fulfil some particular requirements associated with the purpose of the detector and the conditions in the W7-X as well. Therefore, the design of the 'CO Monitor' system allows appropriate adjustment and stabilization of the position of the detectors, using specially designed flanges with stabilizing rods connecting vacuum chamber with detectors. Moreover, it is possible to apply their different types.

In the next sections the detailed characteristics of requirements as well as considered options for the detectors are described.

\section{General requirements for the detector}

For light impurities observation in the XUV/soft X-ray range with hot plasmas experiments, the proper detection systems need to be applied. Due to the fact that system has to cope with some requirements, also the detector should fulfil certain properties. Since the 'CO Monitor' system will possess high throughput and high time resolution (1ms), the detector system should provide the fast measurement and fast processing of the data collected during experiment. The estimated flux of the quanta reaching the detector is close to $10^{9} \mathrm{~s}^{-1} \mathrm{~cm}^{-2}$. After the first phase of operation of the spectrometer and experimental verification of its validity, the diagnostic could become a real-time system included in the safety feedback system of the machine. Such information should be delivered constantly with its best accuracy in order to obtain high throughput. Moreover, such detector ought to have relatively high sensitivity in the wavelength range of the observed lines. The selected spectral lines are well separated, and the spectral resolution of the spectrometer ensures, that only one line is registered by one detector. As the shape of the spectral line will not be studied, the spatial resolution does not need to be very high (sufficient only to distinguish the spectral line core and its far wings).

For the observation of narrow band of $\mathrm{C}, \mathrm{B}, \mathrm{O}$ and $\mathrm{N}$ elements by a spectrometer based on the Johann geometry with bended dispersive elements, it is essential that the detector should have appropriate detection area. As each of the detectors is associated with only one spectral line, its length should be adequate to cover the width of the spectral line imaged to focusing plane of the spectrometer (40 mm). Hence, its height can be comparable to the height of the dispersive element $(20 \mathrm{~mm})$. In order to get signal to noise ratio of at least 10, the quantum efficiency of the detector ought to be as high as possible. Such noise can be associated with the intrinsic properties of the detection system as well as with the influence of the other systems of the W7-X, e.g., ECRH heating. The sensitivity of some of the detectors (e.g., Micro Channel Plates) changes over time of its operation (like on KT4 on JET [8]). It is not crucial in case of qualitative measurements but for obtaining quantitative information the system would require frequent recalibration or cross-calibration with another spectrometer. From the point of view of physical environment of the detector, it has to be taken into account that the equipment is going to work in the magnetic field reaching the level of $75-100 \mathrm{mT}$. The magnetic field, unlike in many other MCF experiments (e.g. JET, ASDEX), will be switched-on constantly for hours (or days). Moreover, in the future experiments which will be performed also with deuterium plasmas the particular increase of neutron yield is predicted. The neutrons can distort the measured signal by direct hitting the detection area and cause some damage of the detector electronics as well as the detector itself. Taking into account all the above it was essential to find the most suitable option for the detector that will be applied for the 'CO Monitor' diagnostic system. An overview consideration on the selection of the detectors has been performed and is presented in the next section.

\section{Detection systems under considerations}

For the measurements of lines in the wavelength ranges of interest (C VI - $3.4 \mathrm{~nm}, \mathrm{O}$ VIII - $1.9 \mathrm{~nm}, \mathrm{~B} \mathrm{~V}-4.9 \mathrm{~nm}$, $\mathrm{N}$ VII $-2.5 \mathrm{~nm}$ ), there are several options possible to be applied. The first option under consideration are Micro Channel Plates (MCPs) which are commonly used on other machines, e.g., in KT4 diagnostic at Joint European Torus (JET). These are composed of MCPs connected to a phosphor screen image intensifiers. The basic principle of operation [9] is multiplication of photoelectrons emitted by the incoming radiation. Once the VUV/XUV photons reach the MCPs, they produce the photoelectrons that are multiplied in the microchannels. Depending on the voltage applied to the MCPs, the particular amplification of emitted photoelectrons (even up to $10^{4}$ ) is achieved. Obtained electrons are subsequently accelerated and focused on the luminofor (phosphor plate) by additional voltage (approx. $3-5 \mathrm{keV}$ ). This voltage also 
enhances the efficiency of energy conversion in the phosphor. The time resolution of such system depends on each component as readout electronics or fluorescence lifetime of the phosphor.

However, there are some drawbacks that need to be taken into account. First of all, the system based on the MCPs has to be constantly under high vacuum (approx.10-6 mbar), since if the vacuum level is deficient the detector may be damaged. This requires some additional safety systems based on the voltage cut off if the pressure suddenly increases and/or individual pumping systems dedicated directly for achieving the proper vacuum level for the MCPs operation (e.g., HEXOS [10]).

Another disadvantage of MCPs is their sensitivity which decreases with time significantly, what was observed at KT4 diagnostic system on JET [8]. Due to that fact, for the long operation purposes the calibration process needs to be cyclically repeated. Moreover, it was observed that after very high energy discharges associated with high photon fluxes at JET, the sensitivity of the system decreases rapidly and recovers to the previous level of sensitivity after several hours.

Another option is to use the commercially available CCD cameras, which is a well-known and developed technology. This solution is commonly used in many other plasma diagnostic systems, e.g., X-ray crystal spectrometer at Large Helical Device (LHD) [11]. In our case, one of the considered options is the Andor Newton SO DO920P camera, which assures high linear response (better than 99\%) in the energy range of interest [12]. An advantage of such solution is its availability and high sensitivity in the area of interest linearly increasing from 60 to $90 \%$.

Another essential advantage is a high time resolution of such equipment. The latest available models assure time resolution better than $1 \mathrm{~ms}$ which fulfills the requirements of its application in the 'CO Monitor' system. The considered model is characterized by full spectrum readout frequency above $1 \mathrm{kHz}$ (approx. $1.3 \mathrm{kHz}$ ), that corresponds to time resolution below $1 \mathrm{~ms}$. The sensitivity of considered CCD camera model is quite high also in the range of visible light, however the reflectivity of the dispersive element does not exceed 5\%. For those reasons, the system would suffer from the high background noise level that can occur during the operation. Also, some possible heat loads on the inner wall of stellarator during operation can cause the sudden increase of background noise level significantly since the sensitivity of such detector is approximately four times higher in the XUV range than to visible light. This in extreme case would saturate the detector and blind the system. In the latter phases of W7-X operation when the Deuterium plasmas are going to be investigated, neutron yield may appear. It is difficult to estimate the neutron resistivity and how long the detectors based on the CCD cameras could withstand such conditions. The solution for that hazard would be an implementation of neutron shielding housing for each of the detectors. Such solution is successfully applied as a protection of neutron-sensitive equipment, e.g., at ASDEX-U.
An alternative for the detectors based on semiconductor sensors can be gas detectors in a type of proportional counter. One of such, position sensitive, gas detector is Multi Strip Gaseous Chambers (MSGC) which is applied, e.g., at ASDEX experiment. The detection system based on proportional counter has been successfully applied in the similar diagnostics - KS6 at JET. The sensitivity of such kind of detector in the selected energy range depends on its construction. The basic principle is that the incident photons reaching the chamber ionizes the working gas which the chamber is filled with - usually Argon with small admixture of quenching gas (e.g., methane). The primary electrons are accelerated in HV electric field and by avalanche amplification create the electrical signal proportional to the deposited photon energy. Low energy photons (e.g., in the visible range) have too little energy to ionize the gas and high energy photons, because of much lower cross-section than XUV photons, can pass through the detector with low probability of any collision inside the chamber. That allows to construct the detector with selective range of energy photons under observation. Moreover, its sensitivity does not decrease over time, thus it was successfully used as a reference diagnostic, e.g., "CO Monitor" on ASDEX-U [13]. One of the main factors which determine the efficiency of the detector is transmission of the detector window (thin foil) which separates the gas filled chamber of the detector from the vacuum area of 'CO Monitor' diagnostic system. Such window would be composed of a thin Mylar foil with $0,9 \mu \mathrm{m}$ thickness with $100 \mathrm{~nm}$ of Aluminium coating preventing emerging of the space charge on the foil surface.

Nevertheless, due to the relatively high pressure gradient (approx. $1 \mathrm{~atm}$ ) between the gas chamber and the vacuum area of the 'CO Monitor', there exists a risk of leakage or even an accident associated with uncontrolled rupture of the window. That would result in sudden increase of the pressure in the 'CO Monitor' vacuum and leakage of the counter gas into the plasma vessel. In order to avoid such hazard, a dedicated safety system with a feedback between the main gate valve and vacuum gauges needs to be installed. Moreover, the MSGC detectors are characterized by quite low amplification factor (approx. 100 ), and dynamic range which could result in necessity to frequent adjusting the incoming radiation flux by means of the variable aperture.

In order to solve those issues, the gas electron multiplier (GEM) detectors could be proposed [14-15]. As they are offering more than one stage of amplifying they are characterized by much higher amplification and dynamic range. GEM detectors also do not suffer from neutron impact as MCPs or CCD cameras, that is why it is considered to be applied especially in the latter phase of operation. Nevertheless, beside the presence of a gas as a working medium and the pressure difference issue that was mentioned as drawbacks of typical proportional counter, the construction of the GEM detectors is much more complicated hence much more expensive.

Taking into account all the previously described features of each detector type it was decided to apply CCD 
cameras for the OP2 due to their availability and high sensitivity in the spectral range of interest. Nevertheless, for the future application, especially for campaigns with deuterium plasmas operation on W7-X (and hence when the neutron yield is expected), other options are considered.

\section{Summary}

The 'CO Monitor' is a new diagnostic system, dedicated for investigation of the light impurities behaviour in the W7-X stellarator. The system is already designed and is in constructional phase. It is planned to be installed at W7$X$ in 2020 and commissioned during OP 2 campaign which will be associated with helium and hydrogen plasmas. It consists of two sub-spectrometers with two spectral channels each, dedicated for the measurement of particular Lyman- $\alpha$ lines - carbon $(3.4 \mathrm{~nm})$, oxygen (1.9 $\mathrm{nm})$, nitrogen $(2.5 \mathrm{~nm})$ and boron $(4.9 \mathrm{~nm})$. Each spectral channel is equipped with separate dispersive element and individual detector. The main purpose will be the constant observation of the selected lines, with high temporal resolution (better than $1 \mathrm{~ms}$ ) but the line shapes are not going to be investigated. The construction of ' $\mathrm{CO}$ Monitor' ensures to apply different types of the detectors which will be more appropriate for deuterium plasmas in the future operational phases (e.g., less vulnerable to neutrons). Taking into account the purpose and the particular requirements that the detectors need to face with, the choice of its proper type is very essential. In this paper the discussion about the detector types that are taken under consideration for the application in the 'CO Monitor' system is presented. Several types of detecting systems, like CCD cameras, MCPs and gas detectors in a type of proportional counters, were presented and their features were described. As the W7-X experiment is very unique (e.g., high energy discharges associated with high photon fluxes and very long pulses), it is impossible to create a test bench simulating working conditions of the detectors. Therefore, the selection of the detector was based on the critical survey of existing possibilities. Nevertheless, an option of its exchange after first phase of operation remains open. The main candidate for the detector type for OP 2 is a CCD camera which is commercially available and which sensitivity in the spectral range of interest reaches up to $90 \%$. Nevertheless, each of the detectors is taken into consideration for the future application when the deuterium plasma experiments will be performed.

\section{Acknowledgments}

This work has been carried out within the framework of the EUROfusion Consortium and has received funding from the Euroatom research and training programme 2014-2018 and 2019-2020 under grant agreement No 633053. The views and opinions expressed herein do not necessarily reflect those of the European Commission. This scientific work was partly supported by Polish Ministry of Science and Higher Education within the framework of the scientific financial resources in the years 2014 - 2019 allocated for the realization of the international co-financed project.

\section{References}

[1] M. Krychowiak et al., Overview of diagnostic performance and results for the first operation phase in Wendelstein 7-X (invited), Rev. Sci. Instrum. 87 (2016) 11D304. doi:10.1063/1.4964376.

[2] R. Koenig, et al., The Set of Diagnostics for the First Operation Campaign of the Wendelstein 7$\mathrm{X}$ Stellarator, Journal of Instrumentation 10 (2015) P10002

[3] H. Thomsen et al., Startup impurity diagnostics in Wendelstein 7-X stellarator in the first operational phase, Journal of Instrumentation 10 (2015) P10015

[4] M. Kubkowska et al., First Results from the Soft X-ray Pulse Height Analysis System on Wendelstein 7-X Stellarator, Fusion Eng. Des. (2017). doi:10.1016/j.fusengdes.2017.12.024.

[5] W. Biel et al., Design of a high-efficiency extreme ultraviolet overview spectrometer system for plasma impurity studies on the stellarator experiment Wendelstein 7-X, Rev. Sci. Instrum. 75 (2004) 3268-3275. doi:10.1063/1.1784557.

[6] N.A. Pablant et al., Layout and results from the initial operation of the high-resolution x-ray imaging crystal spectrometer on the Large Helical Device, Rev. Sci. Instrum. 83 (2012) 083506. doi:10.1063/1.4744935.

[7] G. Bertschinger et al., Compact imaging Bragg spectrometer for fusion devices, Cit. Rev. Sci. Instruments. 75 (2004) 3727. doi:10.1063/1.1781755.

[8] I. Książek, Private Communication, IPPLM, Warsaw, Poland, 2018

[9] J.L. Schwob et al., High-resolution duomultichannel soft x-ray spectrometer for tokamak plasma diagnostics, Rev. Sci. Instrum. 58 (1987) 1601-1615. doi:10.1063/1.1139408.

[10] B. Buttenschön et al., Proceedings of 43rd EPS Conference on Controlled Fusion and Plasma Physics, Leuven, Belgium, 4-8 July 2016, Vol. 40A, P4.012

[11] I. Sakurai et al., Development of a Wide Band and Compact X-Ray Crystal Spectrometer for Iron Charge State Distribution Measurement on LHD, 2 (2007) 1068. doi:10.1585/pfr.2.S1068.

[12] Www.tokyoinst.co.jp/product_file/file/AD08_tec 02_ja.pdf

[13] R. Neu et al., Monitor for the carbon and oxygen impurities ind the ASDEX Upgrade tokamak, 67 (1996) 1829. doi:10.1063/1.1147522

[14] M. Chernyshova et al., Conceptual design and development of GEM based detecting system for tomographic tungsten focused transport monitoring, J. Instrum. 10 (2015) P10022P10022. doi:10.1088/1748-0221/10/10/P10022.

[15] M. Chernyshova et al. Gaseous electron multiplier-based soft x-ray plasma diagnostics development: Preliminary tests at ASDEX Upgrade, Review of Scientific Instruments 87, 
11E325 (2016);

https://doi.org/10.1063/1.4960305. 